\title{
An Interactive Perception Based Model for Characterization of Display Devices
}

\author{
Attila Neumann* Alessandro Artusi* Georg Zotti* László Neumann ${ }^{\dagger}$ Werner Purgathofer* \\ * Institute of Computer Graphics and Algorithms \\ University of Technology, Vienna, Austria \\ email:\{aneumann,artusi,gzotti,wp\}@cg.tuwien.ac.at \\ † Grup de Gràfics de Girona, Universitat de Girona, Spain \\ Institució Catalana de Recerca i Estudis Avançats, ICREA, Barcelona \\ email:Ineumann@ima.udg.es
}

\begin{abstract}
This paper describes a simple to use, yet accurate way to obtain the Tone Reproduction Curve (TRC) of display devices without the use of a measurement device. Human vision is used to compare a series of dithered color patches against interactively changeable homogeneously colored display areas. Results comparing this method with spectrophotometer measurements are given for three monitors.
\end{abstract}

Keywords: Display Measurement, Human Visual System

\section{INTRODUCTION}

Several display characterization models with different characteristics have been presented in the past. These models can be classified into two basic categories: measuring device based and human vision based models. Many works have been presented for the first category, ${ }^{1-6}$ trying to model the real Tone Reproduction Curve (TRC) characteristic of a CRT display device. In many cases these models are still not accurate enough to acquire the real TRC, but just an approximation of it. In addition, even if they reach sufficient accuracy for CRT displays, this accuracy is not achieved for LCD displays. In consequence, the users are not able to have the high precision required for many applications. On the other hand a model for LCD displays has been proposed ${ }^{7}$ which introduces a spline interpolation in order to estimate the TRC.

In any case, these models require a spectrophotometer to get the information necessary to describe the TRC.

The models of the second category are based on interaction with the user and based on human vision and observation. One example is used in commercial software such as Adobe Gamma $^{\mathrm{TM}}$ which comes with Adobe Photoshop ${ }^{\mathrm{TM}}$.

While in the first category acceptable quality can be achieved, in the second one this goal has not been achieved until now for two reasons. First, the current models are not able to estimate or compute the real TRC, but only a simplification of the model used in the first category. Second, the applied mathematical background in these models is typically restricted to describe a kind of simple exponential gamma correction function. A more accurate characterization model of display devices based only on human observation is still required.

In this paper we introduce a novel and accurate characterization model to acquire the TRCs of the color channels of display devices without using a spectrophotometer, based only on human perception and interactive decisions.

The paper is structured as follows: first, an overview of previous work on modeling TRCs is given. The next section explains the advantage of using human perception to compare equal appearance of color patches during a measurement process. The main section describes our method in detail, giving the mathematical background for the TRC acquisition. Results of TRC acquisition with our method in comparison to measurement with a spectrophotometer and calibration with Adobe Gamma ${ }^{\mathrm{TM}}$ for three monitors are given in the next section, followed by our conclusion.

\section{TONE REPRODUCTION CURVE (TRC)}

A CRT display is characterized by an important property that describes the relationship between the input signal of the monitor and the luminance produced on the screen. It is represented by a curve called Tone Reproduction Curve (TRC), also known as Transfer Function (TF). ${ }^{8}$

A simple TRC function is characterized by a parameter called $\gamma$ (gamma). It indicates a relationship between voltage input and light output known to physicists as fivehalves power law, ${ }^{8}$ describing the relation of the intensity $L$ of light produced at the face of the screen and the voltage input $V_{I}: L \sim V_{I}^{2.5}$. The functions associated with the three guns of a color CRT are not necessarily identical. The process used to correct this non-linearity to achieve correct reproduction of intensity is called gamma-correction. ${ }^{8}$ 
On the other hand, in case that high quality color reproduction is required, the complexity of this nonlinearity can not be described just with a simple gamma value (power function). Several theoretical models which try to describe the real TRC curve have been proposed. ${ }^{5}$ These models are also called physical models, in the sense that they put in relation physical parameters of the CRT display.

The simple Gamma model is frequently used and is described by

$$
R\left(d_{i}\right)=a d_{i}^{\gamma} .
$$

The value $d_{i}\left(0 \leq d_{i} \leq 1\right)$ represents the digital input for each channel $i=R, G, B$ of the CRT display. The values $R\left(d_{i}\right)$ are the TRC response. The parameter $a$ is called Gain.

The GOG model (Gain, Offset, Gamma) introduces another parameter $b=1-a$, called offset. ${ }^{4}$ In this model, offset $b$ must be negative, in other words the gain value $a$ must be greater than $1.0^{4}$ :

$$
R\left(d_{i}\right)=\left(a d_{i}+b\right)^{\gamma}
$$

Version 1.x of the IEC model introduces a different TRC curve, called GGO (Gain, Gamma, Offset):

$$
R\left(d_{i}\right)=a d_{i}^{\gamma}+b
$$

Evolutionary development of the IEC model brought versions 2.x and 3.x, also called GOGO (Gain, Offset, Gamma, Offset) which added a second coefficient $c$ (Offset) to the GOG model in order to take internal flare into account when it is not otherwise accounted for ${ }^{4}$ :

$$
R\left(d_{i}\right)=\left(a d_{i}+b\right)^{\gamma}+c .
$$

Also several nonlinear functions to estimate the TRC curve have been proposed in the literature ${ }^{6,9}$ : Polynomials of second order LIN-LIN2 (5), model LOG-LIN (6), model LOGLIN2 (7), model LOG-LOG (8), and model LOG-LOG2 (9).

$$
\begin{aligned}
R\left(d_{i}\right) & =q_{1}+q_{2} d_{i}+q_{3} d_{i}^{2} \\
\log \left(R\left(d_{i}\right)\right) & =q_{1}+q_{2} d_{i} \\
\log \left(R\left(d_{i}\right)\right) & =q_{1}+q_{2} d_{i}+q_{3} d_{i}^{2} \\
\log \left(R\left(d_{i}\right)\right) & =q_{1}+q_{2} \log \left(d_{i}\right) \\
\log \left(R\left(d_{i}\right)\right) & =q_{1}+q_{2} \log \left(d_{i}\right)+q_{3}\left(\log d_{i}\right)^{2}
\end{aligned}
$$

These models are empirical models without relation to the physical characteristics of the CRT display.

All these models are used to describe the relationship between digital input and TRC response for a CRT display. Their accuracy is sufficient for CRT displays, but it is not appropriate for LCD displays. In fact, LCD displays present two main deficiencies as: channel interaction and non-constancy of channel chromaticity. ${ }^{7}$ Other models have been proposed to overcome these deficiencies, e.g. the S-curve model and Masking model.

The $S$-curve model ${ }^{10,11}$ characterizes the relationship between the digital input and the TRC response using the $S$-shaped function defined as

$$
R\left(d_{i}\right)=A_{i R} f\left(d_{i}\right)+A_{i G} f^{\prime}\left(d_{i}\right)+A_{i B} f^{\prime \prime}\left(d_{i}\right)
$$

with weight factors $A_{i j}, i, j=R, G, B, f\left(d_{i}\right)=\frac{d_{i}^{\alpha}}{\left(d_{i}^{\beta}+c\right)}$, and $f^{\prime}$ and $f^{\prime \prime}$ as the first and second derivative of $f$, respectively.

This model considers the channel chromaticity nonconstancy of LCD displays. On the other hand it requires a great deal of training data compared to the other models because of its large number of unknown coefficients. ${ }^{7}$

The Masking and Modified Masking models proposed by Tamura et al. $^{7}$ use a spline interpolation to define the relationship between the digital input and the TRC response They also take into account the two major problems in the colorimetric characterization of an LCD mentioned above. Tamura et al. ${ }^{7}$ showed that these methods are more effective for colorimetric characterization of LCD displays.

However, all the methods presented above require a spectrophotometer to measure a set of data of colors and derive from them the relationship between the input signals and TRC response.

\section{PRINCIPLE}

Characterization of a display based only on human perception has advantages as well as disadvantages. The obvious disadvantage is implied by the adaptation mechanism of human perception, making it impossible to define absolute values: the result of an observation is always depending on its circumstances. Only a direct comparison of two adjacent regions can be reliable, especially the detection of their identical appearance, which is used also by our method.

Fortunately, observations of the apparent identity of two neighbouring color patches will yield constant results for widely changing environmental lighting conditions. It is arguably the only precise human observation, while changing circumstances can drastically change any absolute values. For example, a badly lit white table will still appear white, in spite of its absolute color appearing dark gray. The environmental lighting affects only the accuracy of the observation of emissive color patches, i.e., in case of pure emission the observation is more accurate than in case of added ambient lighting comparable to or brighter than the investigated self emission. 
Equality of two adjacent emissive color patches is observed as disappearance of the visible border between them. Observing the border means observing the existence of contrast between its two sides. The visible contrast is different for different luminance values. According to the law of Weber-Fechner we can roughly distinguish $1 \%$ difference of the background luminance level. Therefore, increasing levels of ambient light will decrease contrast sensitivity, that is, observational accuracy. The deeper character of just noticable contrast is described by spatial vision models and with the contrast sensitivity function (see section 4.1).

Another point is that using inhomogeneous ambient illumination on the area of the display itself, the increase of illumination on the support area of the comparison reduces the accuracy of the comparison, but not its result, since this variation modifies the perceived difference between the two small neighbouring areas only slightly. In addition, when the observer recognizes that the lighting is not uniform, the comparison will undergo a self correction rather than yield a bad result: Since the comparison does not deal with separate border points, but the whole borderline, equality will be perceived when all "trustworthy" border sections seem attenuated. This means, the observer automatically ignores the parts of the border where the non-uniform ambient illumination appears to be changing too rapidly. Any difference in the remaining, "valid" part of the display will be visible and noted by the observer.

Commercial software sometimes includes applets which allow to find the gamma value of the display based on the same principle, so our method can be seen as an extension of them. All the TRCs yielded by this method can be seen only as relative, depending on their maximum and minimum values, so they need a few additional measurements to complete them by these absolute values. However, for many applications it is sufficient to know the relative curves, so the real question is the accuracy that can be reached with our method, compared to an instrument-based method. Now, the advantage of this method is, its accuracy corresponds to that of human perception, which cannot be asserted for all the measuring devices used for such purposes. In other words, we can achieve just the required accuracy if the same environment is given during the measurement process and during some correction operation depending on its results. In addition, it is easy to choose more appropriate circumstances for the measurement process, e.g., a dark environment and observation and judgement by more persons, which results in more accurate curves.

In contrary of the above mentioned definition of a single value (gamma) as the exponent of a power function, our methology uses several elementary measurements with different parameters but by the same basic process for all three color channels.

This basic measurement is perfomed by tuning a singular color value of a homogeneous display area and visually comparing it to another, fixed, non-homogeneous area which combines two given color values by dithering with a given ratio. This step yields a singular signal value for the two input colors and their mixing ratio, that is, such an elementary measurement defines a proportional triplet on the range of the unknown TRC of one of the color channels. During the whole measurement process, appropriate sets of these triplets can be obtained for all three color channels. The 'next triplet' and the setup of the 'next measurement' can be defined depending on or independent of the results of the already existing data. To complete the process, a data analysis and optimization step computes the curve in question, which can be specified by combining certain criteria like the minimization of the differences at each point or the overall smoothness of the resulting curve.

The criteria of the overall evaluation of the measurements as well as the definition of their series can be controlled by different criteria such as minimizing the number of measurements, having a fixed procedure or gaining the most exact characterization of the display possible.

\section{METHOD}

\subsection{Basics}

The method deals with color channels separately. Consequently the repeated computation is used only on onedimensional problems, independent from each other. We ignore the by-densities and the cross effects between the $r$, $g$ and $b$ color channels. In fact, for CRT the cross effect is 1-3\%. In lack of other colorimetric information we assume that the CIE xy chromacity values are according the sRGB recommendation, the ITU-R BT.709 standard: (0.64, 0.33), $(0.30,0.60)$ and $(0.15,0.06)$ for the $r, g$, and $b$ channels, and D65 as white point with values $(0.3127,0.3290)$. As mentioned in the previous section, only visual comparisons are used as relative inputs, so the result is also a relative function which describes the relative luminance values of the independent channels, from 0 to 255 . The relative luminance values $l_{r}, l_{g}, l_{b}$ can be converted to absolute ones $L_{r}$, $L_{g}, L_{b}$ by

$$
L_{c}(v a l)=L_{c}(0)+\frac{L_{c}(255)-L_{c}(0)}{l_{c}(255)-l_{c}(0)} \cdot l_{c}(v a l)
$$

where $c=r, g, b$ and $v a l=0 \ldots 255$. For the sake of simplicity we will work with $l_{c}(0)=0$ and $l_{c}(255)=1$, so the formula is simplified to

$$
L_{c}(v a l)=L_{c}(0) \cdot\left(1+\left(\frac{L_{c}(255)}{L_{c}(0)}-1\right) \cdot l_{c}(v a l)\right)
$$




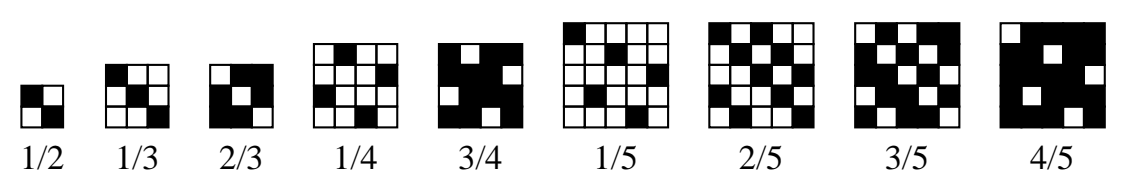

Figure 1: Dither patterns for the "chess-board"

The measured and computed values $l_{c}(\mathrm{val})$ are independent of the absolute values $L_{c}(0)$ and $L_{c}(255)$, and the so also independent of the contrast value $L_{c}(255) / L_{c}(0)$. However, they can be interesting for the overall appearance, but finding them is outside of this method.

This method with only human observation can not determine the absolute contrast, i.e., the ratio of $\mathrm{max} / \mathrm{min}$ output luminance levels. With other "calibration applets" based on just-noticeable contrast, using visually equidistant gray series we can estimate a rough value for this contrast, but this is not studied in this paper. We recommend a practical approach to calibrate this singular free parameter after applying the mathematical model described below: The practical range of the perceptual contrast of a typical monitor is $20 \ldots 100$. We can generate sharp and colorful test images with different contrast values in this range using the results of our model. Observing these images simultaneously on the screen, we can select the best one, which contains the most details in the dark regions. We can select for further use the otherwise unknown contrast value of the selected best image.

The core of the comparison process is a single comparison of an area uniformly filled by a single color against another area, the "chess-board", filled by a repeating pixel pattern. The pattern contains only two definite colors or luminance levels low and high), assembled according to a simple, fixed ratio, e.g. $1 / 2$ or some other rational number with small denominator (Figure 1). The homogeneous area encloses the chess-board, acting as background with luminance back.

To be usable, the patterns which are to be observed must appear homogeneous, otherwise the inhomogenity disturbs our observation of its equivalence with a homogeneous area. The spatial vision models give us quantitative values for the required viewing distance.

The contrast sensitivity function (Fig. 2) describes the spatial behavior of our vision. ${ }^{12-15}$ At lowest contrast the human visual system can distinguish about 8 cycles/degree. Let us represent 1 cycle as a pixel pair, i.e., a 2 pixel distance on the screen.

More interesting for us is the reciprocal of this value showing us the visible contrast threshold (Fig. 3). At about 60 cycles/degree a chess-board pattern with the contrast

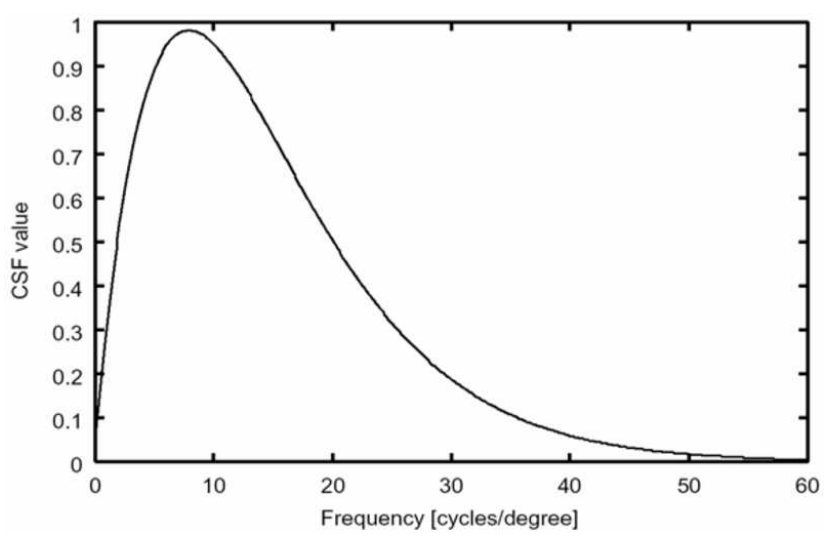

Figure 2: Contrast Sensitivity Function (CSF)

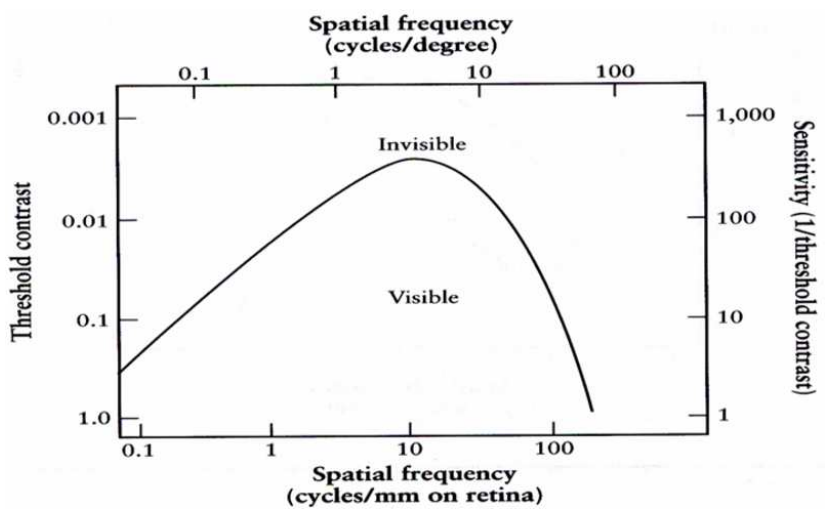

Figure 3: Visible Contrast Threshold 
1 : 100 appears absolute homogeneously gray. We use different patterns, but we can give as example for view distance computation a display with a pixel resolution of 1200 horizontally, from which, with the requirement of $60 \mathrm{cy}-$ cles/degree $=120$ pixels/degree, results that we have to see the display under 10 degrees horizontal view angle. The absolute size of the screen this way determines the required view distance.

Having a pattern, realized by luminances low and high, the observer is requested to tune the third homogeneous luminance level (back) until the luminance difference disappears. Now we have 3 values (low, high, back), and a ratio of the number of the low luminance level pixels within the pattern, ratio $=\frac{N_{\text {low }}}{N_{\text {low }}+N_{\text {high }}}$. The following approximation can be written for the absolute luminance values

$$
L_{c}(\text { back }) \approx L_{c}(\text { low }) \cdot \text { ratio }+L_{c}(\text { high }) \cdot(1-\text { ratio })
$$

for the channels $c=r, g, b$. Using equation (11):

$$
\begin{aligned}
L_{c}(0)+ & Q \cdot l_{c}(\text { back }) \approx \\
& \left(L_{c}(0)+Q \cdot l_{c}(\text { low })\right) \cdot \text { ratio } \\
+\quad & \left(L_{c}(0)+Q \cdot l_{c}(\text { high })\right) \cdot(1-\text { ratio })
\end{aligned}
$$

with $Q=\frac{L_{c}(255)-L_{c}(0)}{l_{c}(255)-l_{c}(0)}$, and reordering

$$
l_{c}(\text { back }) \approx l_{c}(\text { low }) \cdot \text { ratio }+l_{c}(\text { high }) \cdot(1-\text { ratio })
$$

shows the independence of the measurements from the linear calibration, and also that $l_{c}(0)$ and $l_{c}(255)$ can be predefined arbitrarily:

$$
l_{c}(0)=0 \quad \text { and } \quad l_{c}(255)=1
$$

A single measurement gives a single back value for a (low, high, ratio) triplet which can be taken as input for the measurement. back, low and high are bytes, and ratio is a simple rational number. The goal is to define the function $f=l_{c}$, that is, 256 separate values for inputs $0 \ldots 255$.

We now face two mathematical problems

1. Having a list of measurements, a curve is to be defined

2. Having a list of measurements and perhaps a preliminary curve defined by them, either define the next measurement's setup, and/or recommend to stop the process

\subsection{A quadratic minimum problem}

To answer the first question, a principle has to be defined which the function has to fulfill. A set of approximations as in eq. (15) is obviously not enough. The resulting function should fulfill certain conditions, defining its general behaviour.

For the present work we used the simple smoothness condition, more exactly, the minimization of its second derivative. For this, only a finite difference form can be given, that is

$$
S(i)=f(i+1)+f(i-1)-2 f(i) \quad(i=1 \ldots 254)
$$

We transform the approximations (15) for the $N$ measurements:

$$
\begin{aligned}
M(j) & =f\left(\text { low }_{j}\right) \cdot \text { ratio }_{j} \\
+ & f\left(\text { high }_{j}\right) \cdot\left(1-\text { ratio }_{j}\right) \\
& -f\left(\text { back }_{j}\right) \\
\text { where } & (j=1 \ldots N)
\end{aligned}
$$

There are two different approaches considering the measurements' conditions. One is to take them as constraints, i.e., $M(j)=0$, the other is to minimize them together with the other conditions.

It can be argued that there is no exact measurement, at least because setting their values should give an exact real number, but they can be choosen only from a discrete set of numbers. On the other hand, the user can introduce errors by his/her estimation as well, so in addition there can even be more or less contradictional conditions. The problem is solved by a compromise, that is, we solve the compound minimum problem, putting the smoothness conditions and the measurement conditions together, and their importances are accounted for by weight factors $s_{i}$ and $m_{j}$. The optimal result would have all of the $S(i) \quad(i=1 \ldots 254)$ and $M(j) \quad(j=1 \ldots N)$ expressions equal to zero, so we have to minimize the expression

$$
F=\sum_{i=1}^{254} s_{i} \cdot S(i)^{2}+\sum_{j=1}^{N} m_{j} \cdot M(j)^{2}
$$

where by eq. (16) $f(0)$ and $f(1)$ are taken constant, and $F$ is a 254-variable function. As a result we get a smooth function conforming well to the measurements, as expected. All in all there are $256-2+N$ minimum criteria and 2 constraints despite the original 256 variables. These obviously cannot be made zero at the same time, so the solution will be a compromise depending on the weights and the content of $M(j)$.

There are several efficient methods to solve the quadratic minimum problem, the two widest known are mentioned here. One is using an equation system by deriving $F$ mathematically by its variables, which leads to a system of 254 
linear equations with a sparse matrix. The other is directly solving the minimum problem by some of the descent methods. ${ }^{16}$

We have choosen a conjugate gradient method, which in our case is faster by one magnitude than the normal steepest descent (gradient) method. An optimization needs 20-50 elementary steps, each of them consisting of an evaluation of $F$ and its derivative.

A problem not yet mentioned is the definition of the constants $s_{i}$ and $m_{i}$. Considering the equal importance of the different smoothness conditions and the different measurement conditions respectively, we took $s_{i}=s$ and $m_{j}=m$. Multiplying the whole problem by a constant we can eliminate the $s$, that is $s=1$ can be assumed, so it is enough to define $m$.

To define this value, let us consider the overall behaviour of the solution. Optimizing of the total smoothness leads to spline-like curves, where the magnitude of the average value of the second derivative is $1 / 255^{2}$, and its overall sum is $1 / 255$. Minimizing its distribution, we get a slowly changing second derivative, i.e., a curve behaving locally similar to a polynome of about 3rd degree. A sudden jump causes an $O(1)$ constant anomaly, so if the magnitude of $m$ is between 1 and $1 / 255$, e.g. $1 / \sqrt{255}$, we have a locally and also globally well behaving function. Of course this value can be modified or tuned further by demand.

\subsection{Power function as a non-convex solution}

Considering that the optimization introduced above tries to reach maximal smoothness which leads to functions behaving locally like 3rd order polynoms, and also considering that the display characteristics used to be approximately a power function which differs from this one, another approach was investigated too.

We could transform the coordinate system, the domain as well as the range, to a log-log scale so that

$$
\log y=g(\log (x / 255)) \quad \text { when } \quad y=f(x)
$$

Linear functions of this coordinate system are corresponding to $c \cdot x^{p}$ power functions in the original system, and the minimum problem results in the possibly smoothest functions, that is, the functions most similar to linear functions, so this transformation looks like the appropriate way to get power-like functions in the original coordinate system.

Taking the new variables, the coefficients of the smoothness conditions will change, and also the measurement conditions shall be rewritten by the exp functions of the new variables, since the formula is applicable on the original values. All in all another minimum problem is to be solved.
Unfortunately the coefficients of the new problem have very different magnitudes, the biggest ratio between them is about $1: 2^{32}$.

In addition, the function in question is not purely quadratic, and it is not even convex. These facts lead to inconveniences, either in the numerical stability of the algorithm or in its correctness, when the components of the function are overweighted in order to get rid of the numerical instability. This question needs further research.

\subsection{The stop criterium}

The measurement shall be exact and at the same time the number of the measurements should be minimized to achieve good results with low effort for the user. These requirements lead to another optimization problem: defining the next measurement step in an optimal way, and notifying the user when reliability has reached a certain limit.

The main problem for answering the question about the next step is that the expected effect of a next measurement on the reliability of the function should be evaluated depending on the behaviour of the still unknown function itself. How could this function behave between the points (byte values of colors) where we already have some information, and how can we rely on the previous measurements? These questions are connected to the principles of the optimization method. We used some heuristics relying on experiments, in order to get a compromise between the simplicity and accuracy, where the observer can overrule the recommendation to stop.

A measurement means a definition of a value by the user, where the minimum (low) and maximum ( $h i g h$ ) values are given as well as their mixing ratio (ratio), represented by a pattern. Then the low, high and also the resulting intermediate point would be taken as support points. The method restricts the possible low and high points for the new measurement only on the set of already used support points. The central players of the process are the Reliability and its opposite and counterpart, the Uncertainty. The simple rule is that the Reliabilities are to be added, its meaning and usability is of course depending on the content of the Reliability.

The process defining the next measurement consists of three successive steps.

1. Two kinds or layers of Reliabilities are defined. First the existing support points' reliabilities are defined as the sum of all the Reliabilities coming from the measurements in which these points appear (as low, high, or medium point). The points 0 and 255 are naturally assigned absolute Reliabilities (i.e. zero Uncertainty). 
An individual measurement's Uncertainty is equal to the error of its condition (19) to be minimized, multiplied by a factor representing the possible quality of the measurement in question and consisting of two further factors expressing the effect of the pattern's nonhomogenity and the contrast sensitivity belonging to the luminance to be set.

2. Then, in a technical intermediate step, each point is assigned the other kind of Reliability as a sum of the effects of the support points on the regular point in question. These 'effects' are also some sort of Reliabilities, but directly Uncertainties are computed by adding the Uncertainty of the support point in question and another Uncertainty which is a square function of the distance between the two points. This value characterizes an exisiting set of measurements, so when their compound value, actually their maximum, reaches a certain threshold, the process is suggested to be terminated.

3. Finally all the possible triplets (low luminance - high luminance pairs with reasonable patterns) are assessed so that the Reliabilities of the regular points are completed by the estimated effect of the triplet, and then their maximum values are compared in order to select the triplet having the smallest of them. To estimate the effect of an incomplete measurement series on a regular point, in order to compute the added Reliability, Uncertainty is computed as a product of the possible quality of the measurement and a sum of the Uncertainties of the given low and high points and the distance dependent Uncertainty.

The main thought of the process is that the effect of an individual (existing or tentative) measurement is computed as sum of Uncertainties, since the effect of the different measurements are added as Reliabilities. Some heuristics are used, such as the computation of the distance dependent Uncertainty, the 'inhomogenity' related to the contrast sensitivity as well as using the maximum norm for unifying the individual Uncertainties and their limit value. All the new measurements' base points are selected from the existing support points, that is, from 0,255 and the previously defined intermediate points, because it makes the process faster and the estimate more reliable.

\section{RESULTS}

We compared our methodology with results from direct measurements of the TRC curve using a GretagMacbeth Spectrolino spectrophotometer, and with results obtained with the Adobe Gamma ${ }^{\mathrm{TM}}$ applet. The experiments are performed on three different monitors, two CRT and one LCD display, named respectively: CRT Nokia Multigraph 445Xpro, CRT SGI GDM-20E21, and LCD EIZO FlexScan L685. The color patterns used to perform the measurements with the Spectrolino were 52 color images per channel with color values 0 to 255 increasing in equal steps. We first compared our results with the TRC curve obtained for the three channels by Adobe Gamma, and the results are shown in figures 4 and 5 .

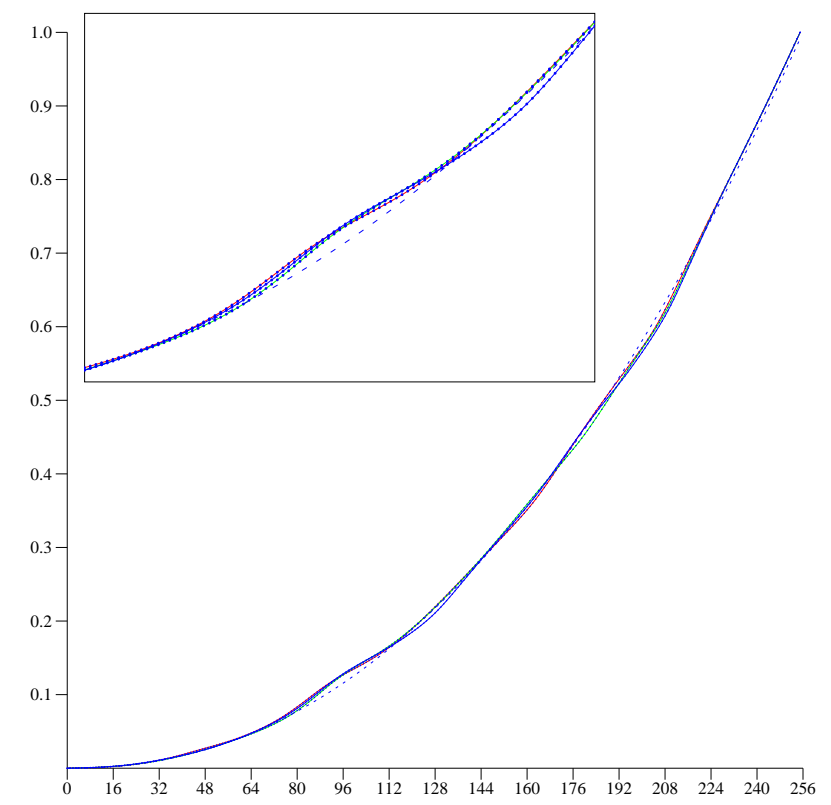

Figure 4. Gamma curve for $\gamma=2.2$ (dashed) and the three color channels for NOKIA Multigraph 445Xpro CRT monitor. Note that all 3 curves are above the gamma curve near $x=96$, and all are below it near $x=215$.

We can observe how the TRC curve obtained with Adobe Gamma is apparently similar to the TRC curve obtained with our method. But a closer observation shows that in reality there are several discrepancies between the TRCs obtained with the two techniques. Both figures show a closeup of two areas where the two TRC curves differ significantly from each other. In order to better understand these differences we produced figure 6 for the red channel of the NOKIA display, which shows the relative difference of the values $(R D V)$ (continuous line) and the difference of derivates $(D D)$ (dashed line). We can observe that for this channel $\max (R D V)$ is about $10 \%$ and $\max (D D)$ is about $30 \%$. This high difference can be perceived by a human observer. Similar results are obtained also for the other two channels (green and blue) of the NOKIA monitor. Results of a equivalent comparison made for the red channel of the SGI CRT monitor are shown in figure 7. 


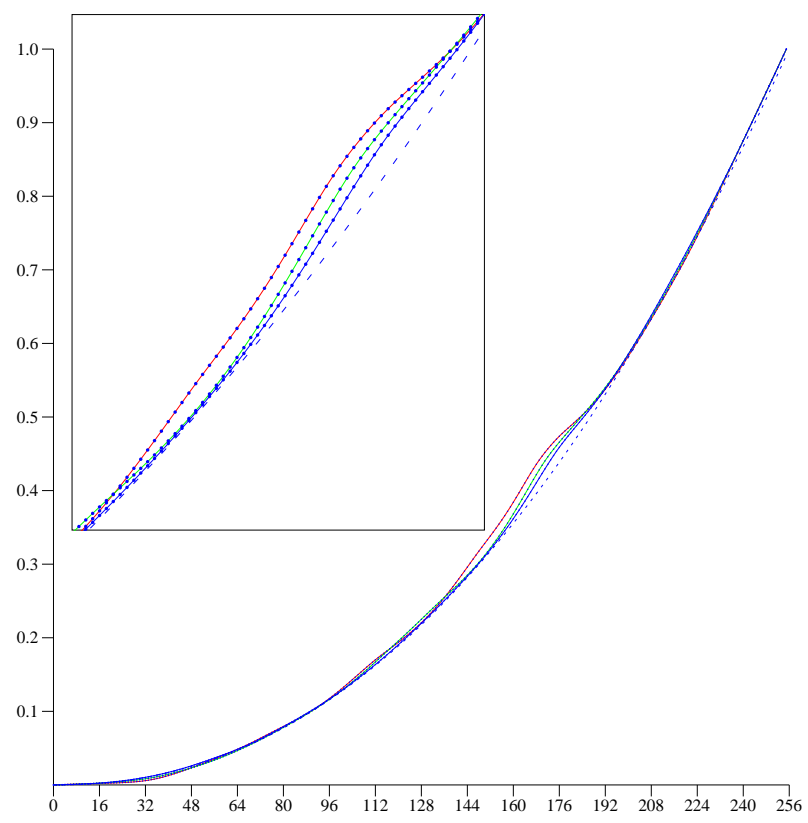

Figure 5. Gamma curve for $\gamma=2.2$ (dashed) and the three color channels for SGI DMG-20E21 CRT monitor. Note that all 3 curves are above the gamma curve near $x=180$.

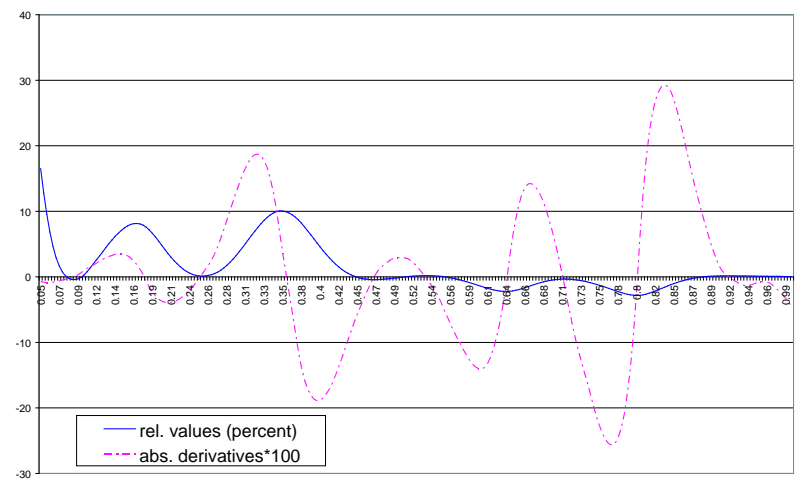

Figure 6. Comparison of red channel for NOKIA monitor with result from Adobe Gamma

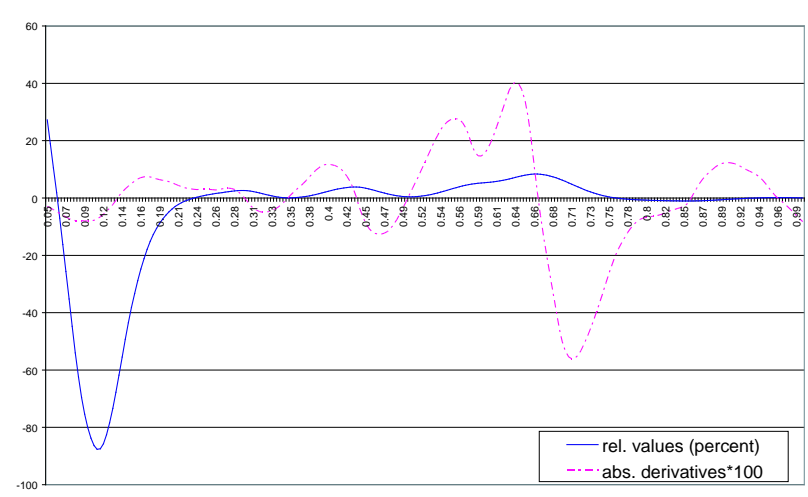

Figure 7. Comparison of red channel for SGI monitor with result from Adobe Gamma

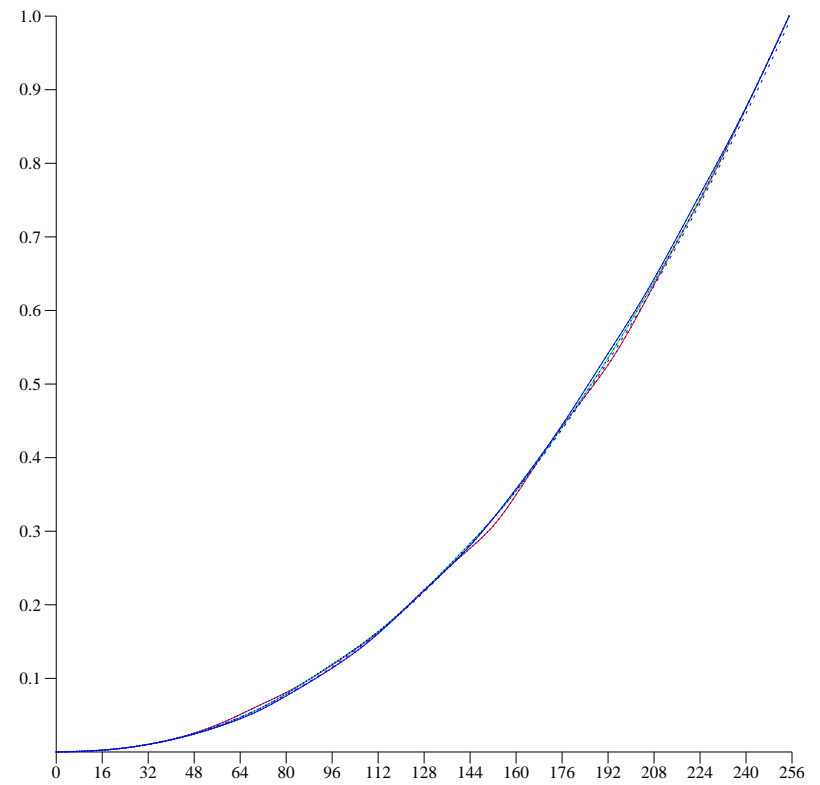

Figure 8. Gamma curve for $\gamma=2.2$ (dashed) and the three color channels for EIZO FlexScan L685 LCD monitor. 


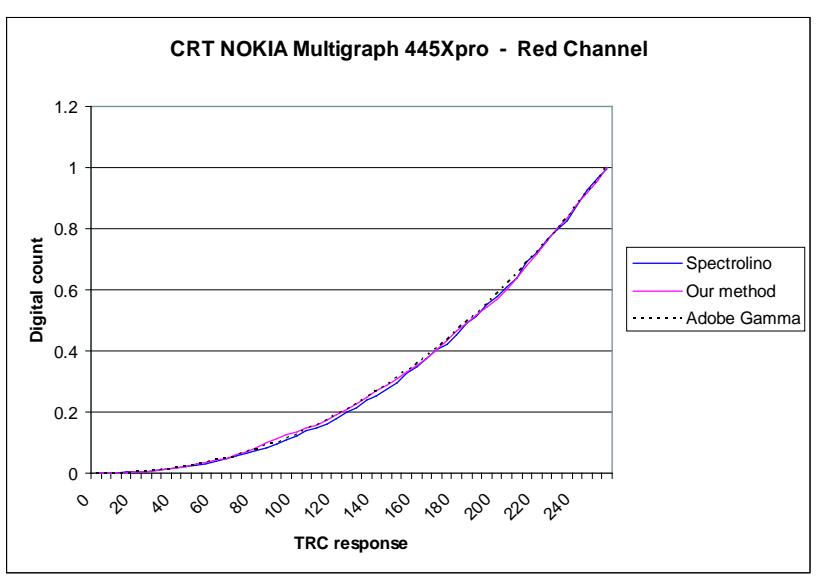

Figure 9. Comparison of red channel for NOKIA monitor with Spectrolino measurements

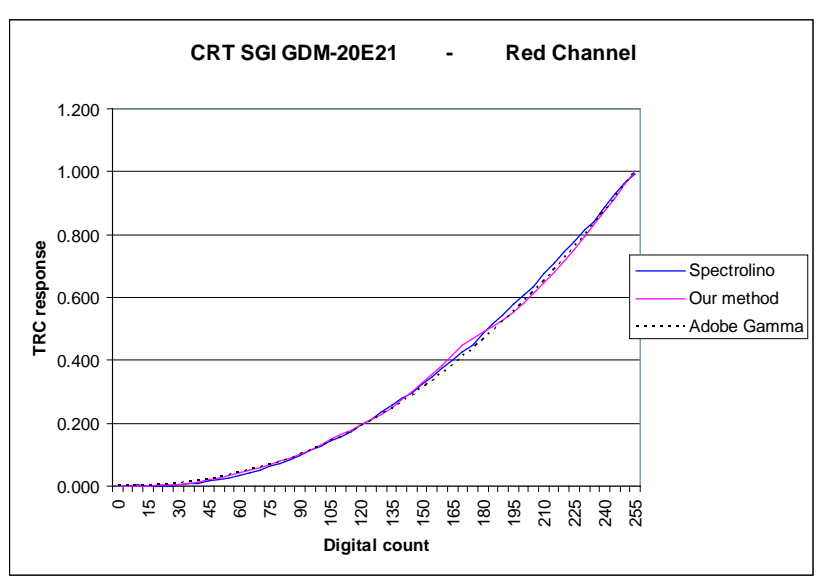

Figure 10. Comparison of red channel for SGI monitor with Spectrolino measurements

A similar observation can be done also for the EIZO LCD monitor, and the graph that reports the discrepancies between the TRC obtained with our method and Adobe Gamma is shown in figure 8.

Our second comparison was done against direct measurements of the TRC curve with a spectrophotometer. The results for the red channel are shown in figures 9, 10 and 11 for all three monitors. In these figures the TRC curve obtained with our method is close to the TRC curve obtained with the spectrophotometer, providing the efficiency of our method.

\section{CONCLUSION}

A novel model has been presented in this paper which is able to perform the characterization of the luminance values of a display. In particular it is able to obtain a relative

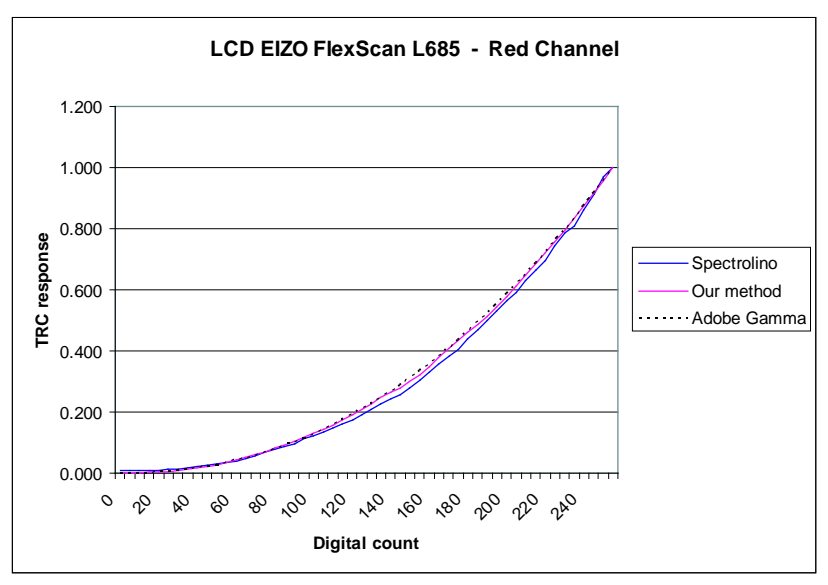

Figure 11. Comparison of red channel for EIZO monitor with Spectrolino measurements

TRC characteristic. Having an approximate contrast value, it is enough information about the display for most of the tone mapping methods. Integration of the model with a tone mapping algorithm is subject of future works.

The flexibility of the model allows it to be used in many applications without the necessity to use a spectrophotometer. Also a fast and simple recharacterization is possible, just using the interactive process with the end user. The main benefit of this approach is that is is a cheap and simple solution, either to define a relative TRC of a display or to verify a given one. The resulting curves are expected to be close to a simple power function, which could be defined also by other means, but the deviations from these curves can be detected as well. In fact their measure and effect can be above the perceptually observable limit, which makes the question and the solution reasonable.

There are a couple of open possibilities in the method. On one hand its usability could be improved by taking information on the absolute values, that is, about the contrast value and the smallest (or the largest) value of the absolute luminance, either staying at the human perception based input or using other methods.

On the other hand its mathematical engine can be improved by different aspects. First by simplifying and making it more reliable by changing the simple smoothness principle to a log-log scale based one, solving the problem of instability and unequal weighting as mentioned in section 4.3. Second by improving the automatic selection of the 'next step', taking any internal coherence and any possible human errors also into account. Third, to have a theoretically and practically better trade-off between the smoothness and observation conditions, since the method depends on human observations and so is affected by possible hu- 
man mistakes. So estimating and correcting its effect can also improve the reliability of the results of the method.

\section{ACKNOWLEDGMENTS}

We would like to thank Benjamin Roch and Wolfgang Deix for their efforts in software development.

This work was partially supported by the European Union within the scope of the RealReflect project IST-200134744, "Realtime Visualization of Complex Reflectance Behaviour in Virtual Prototyping" and by the Spanish Government by project number TIC2001-2416-C03-01.

\section{REFERENCES}

1. R. S. Berns, R. J. Motta, and M. E. Gorzynski, "CRT colorimetry - Part I: Theory and practice," Color. Res. Appl. 8, pp. 299-314, Oct. 1993.

2. R. S. Berns, R. J. Motta, and M. E. Gorzynski, "CRT colorimetry - Part II: Metrology,” Color. Res. Appl. 8, pp. 315-325, Oct. 1993.

3. M. D. Fairchild and D. Wyble, "Colorimetric characterization of the apple studio display (flat panel LCD)," tech. rep., Munsell Color Science Lab., Rochester Institute of Technology, Rochester, NY, July 1998.

4. E. Day, "Colorimetric Characterization of a Computer Controlled (SGI) CRT Display," tech. rep., Munsell Color Science Lab., Rochester Institute of Technology, Rochester, NY, April 2002.

5. N. Kato and T. Deguchi, "Reconsideration of CRT Monitor Characteristics," in Color Imaging: Device Independent Color, Color Hardcopy, and Graphics Arts III, pp. 33-40, 1998.

6. D. L. Post and C. S. Calhoun, "An Evaluation of Methods for Producing Desired Colors on CRT Monitors," Color. Res. Appl. 14, Aug. 1989.

7. N. Tamura, N. Tsumura, and Y. Miyake, "Masking Model for Accurate Colorimetric Characterization of LCD," Journal of the SID 11(2), pp. 1-7, 2003.

8. C. A. Poynton, “Gamma' and its Disguises: The Nonlinear Mappings of Intensity in Perception, CRTs, Film and Video," SMPTE, pp. 1099-1108, Dec. 1993.

9. H. R. Khang, Color Technology for Electronic Imaging Devices, Peerless Corp., 1997.

10. Y. Kwak and L. W. MacDonald, "Accurate Prediction of Colours on Liquid Crystal Displays," in Proc. IS\&T/SID Ninth Color Imaging Conf., pp. 355-359, November 2001.
11. Y. Miyake, H. Saitoh, H. Yaguchi, and N. Tsukada, "Facial Pattern Detection and Color Correction from Television Picture for Newspaper Printing," J. Imag. Technol. 16, pp. 165-169, October 1990.

12. R. L. D. Valois and K. K. D. Valois, Spatial Vision, no. 14 in Oxford Psychology Series, Oxford Univ Press, September 1990.

13. J. Schirillo and S. Shevell, "Brightness Contrast from Inhomogeneous Surrounds," Vision Research 36(12), pp. 1783-1796, 1996.

14. K. Tiippana and R. Nasanen, "Spatial-frequency bandwidth of perceived contrast," Vision Research 39(20), pp. 3399-3403, 1999.

15. A. Watson and C. Ramirez, "A Standard Observer for Spatial Vision." http://vision.arc.nasa. gov/publications/watson_arvo2000. pdf.

16. R. Fletcher, Practical Methods of Optimization, John Wiley \& Sons, 2nd ed., 2001. 